\title{
Is There a Need for One Health Surveillance (OHS)?
}

\author{
John Berezowski ${ }^{* 1}$, Judy Akkina ${ }^{2}$, Victor D. Vilas ${ }^{3}$, Katrina DeVore ${ }^{4}$, Fernanda C. \\ Dórea $^{5}$, Céline Dupuy ${ }^{6}$, Melody J. Maxwell ${ }^{3}$, Vivek Singh ${ }^{7}$, Flavie Vial ${ }^{1}$ and Laura \\ Streichert ${ }^{4}$
}

1University of Bern, Bern, Switzerland; 2United States Department of Agriculture, Animal and Plant Health Inspection Service, Veterinary Services, Fort Collins,, CO, USA; ${ }^{3}$ Pan American Center for Foot-and-Mouth Disease, Pan American Health Organization, Rio de Janeiro, Brazil; ${ }^{4}$ International Society for Disease Surveillance, Boston, MA, USA; ${ }^{5}$ Swedish Zoonosis Centre, Department of Disease Control and Epidemiology, National Veterinary Institute (SVA), Uppsala, Sweden; ${ }^{6}$ French Ministry of Agriculture, Lyon, France; ${ }^{7}$ Public Health Foundation of India (PHFI), Indian Institute of Public Health (IIPH), Hyderabad, India

\section{Objective}

The primary purpose of this study was to explore the attitudes of surveillance stakeholders from different domains to:

-determine whether there is a perceived need for OHS

-identify significant surveillance gaps

-assess the motivation to change (fill the gaps)

A secondary purpose was to gather a group of surveillance stakeholders to identify and prioritize strategies to move One Health Surveillance forward.

\section{Introduction}

As interest in One Health $(\mathrm{OH})$ continues to grow, alternative surveillance infrastructure may be needed to support it(1). Since most population health surveillance is domain specific; as opposed to $\mathrm{OH}$ which crosses multiple domains, changes to surveillance infrastructure may be required to optimize $\mathrm{OH}$ practice. For change to occur there must be a strong motivation that propagates from a perceived need. Since the purpose of surveillance is to produce information to support decision making, the motivation for change should relate to a lack of surveillance information needed to make $\mathrm{OH}$ decisions, or a gap in the surveillance infrastructure required to produce the information (2).

\section{Methods}

The study had two parts:

1) An electronic questionnaire emailed to surveillance stakeholders working in different health domains around the world, to assess their attitudes towards integrated OHS.

Questions included:

-Would OHS be a benefit in your jurisdiction?

-Is OHS a challenge in your jurisdiction?

-How difficult would it be to make improvements in OHS?

-What priority does OHS have in your jurisdiction?

2) A workshop was held at the Annual Conference of the International Society for Diseases Surveillance (ISDS) held in Philadelphia, PA, on Dec 10-11, 2014, where a formal identification and prioritization of solutions to OHS implementation was conducted.

\section{Results}

A total of 185 questionnaires were returned by respondents from 44 countries including low income countries $(58,31 \%)$ and high income countries $(127,69 \%)$. Respondents reported working primarily in public health $(96,52 \%)$, followed by animal health $(36,19 \%)$ and environmental health (4, 2\%). Forty six $(25 \%)$ respondents reported working in multiple domains.

The majority $(158,85 \%)$ of respondents reported that OHS would benefit them in their work. There were no significant differences in the perceived need for OHS across domains, or between low and high income countries.

Almost half of the respondents $(87,47 \%)$ reported that assessing the risk of transmission across domains was a frequent or ever present challenge indicating that a gap in surveillance may exist in some jurisdictions. Most $(129,70 \%)$ reported that improvements would be somewhat difficult or very difficult. Many $(120,65 \%)$ reported that making improvements was a medium to high priority indicating that some respondents were motivated to change.

There were 61 workshop participants from 6 countries who identified solutions, including: cross domain staff exchanges, tools for data integration, and making diseases that require multi-domain collaborative responses or control programs reportable in multiple domains.

\section{Conclusions}

This study provides some support that among surveillance stakeholders, OHS is valued. It also provides support for the existence of surveillance gaps, and the presence of motivation among some stakeholders to improve existing surveillance to meet the information needs of $\mathrm{OH}$.

The study population included respondents who worked in public health, animal health and multiple domains, and from many countries. However the sample was not large and it is not known how well this sample represents the biosurveillance community in general.

Keywords

One Health Surveillance; One Health; Integrated surveillance

\section{Acknowledgments}

This work was supported by the ISDS: http://www.syndromic.org/ and the Skoll Global Threats Fund: http://www.skollglobalthreats.org/

\section{References}

1.Zinsstag, J., Schelling, E., Waltner-Toews, D., Whittaker, M., \& Tanner, M. (2015). One Health: Theory and Practice of Integrated health Approaches. Oxfordshire, UK: CABI.

2.El Allaki, F., Bigras-Poulin, M., Michel, P., \& Ravel, A. (2012). A population health surveillance theory. Epidemiology and Health, 34, e2012007. doi:10.4178/epih/e2012007

\section{*John Berezowski}

E-mail: john.berezowski@gmail.com 